apuntesuniversitarios.upeu.edu.pe

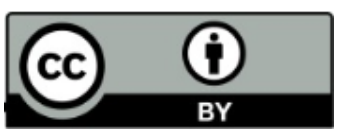

$C C-B Y$

Apuntes Universitarios, 2021: 11(3), julio-setiembre

ISSN: 2304-0335 DOI:https://doi.org/10.17162/au.v11i3.698

\title{
Una huella a través del tiempo: Trascendencia académica desde la práctica docente universitaria
}

\section{A mark through Time: academic transcendence from university teaching practice}

\author{
Carlos Mario Morales Zárate ${ }^{1}$; Carlos Arturo Olarte Ramos, ${ }^{2 a}$ \\ Universidad Juárez Autónoma de Tabasco ${ }^{12}$ \\ (iD) Orcid ID: https://orcid.org/0000-0003-3474-6882 ${ }^{1}$ \\ (iD) Orcid ID: https://orcid.org/0000-0002-9534-51532
}

Recibido:01 de febrero de 2021

Aceptado: 28 de marzo de 2021

\section{Resumen}

La educación contemporánea pretende dejar atrás los modelos tradicionales de enseñanza, donde dominaba quien estaba enfrente del estudiantado; actualmente se favorece la participación bilateral para la construcción de saberes. Este artículo tiene el objetivo de analizar la percepción que el profesorado de educación superior tiene de su función en la enseñanza y cómo su práctica influye en la vida de su alumnado; para ello se desarrolló una investigación cualitativa con enfoque fenomenológico, en la cual se realizaron entrevistas durante el segundo semestre de 2020, a un grupo de docentes de una institución superior que se localiza en el estado mexicano de Tabasco. Se identificó que para trascender se requiere conocimiento en el área, vocación de servicio y empatía, con los que el cuerpo docente responde a las exigencias profesionales que le demandan sus estudiantes y la institución en la que colabora. Se concluye que la trascendencia se vuelve subjetiva, ya que su significación dependerá no solo de lo que piensan y hagan quienes están frente al grupo, sino de quienes reciben la enseñanza.

Palabras clave: Formación docente, trabajo docente, buenas prácticas educativas, calidad de la educación, cualidades del profesorado

\begin{abstract}
Contemporary education pretends to leave behind traditional teaching models, where the person in front of the student body dominated the classroom; currently, bilateral participation is favoured for the construction of knowledge. This article aims to analyse the perception that higher education teachers have of their role in teaching and how their practice influences the lives of their students;
\end{abstract}

${ }^{\text {a} C o r r e s p o n d e n c i a ~ a l ~ a u t o r: ~}$

olarte4@hotmail.com 
for this, a qualitative research with a phenomenological approach was carried out, in which some interviews were conducted during the second semester of 2020, with a group of teachers from a higher institution located in the Mexican state of Tabasco. It was identified that in order to transcend, knowledge in the area, a vocation for service and empathy are required, with which the teaching body makes commitments and responds to the professional demands of its students and the institution in which it collaborates. It is concluded that transcendence becomes subjective, since its significance will depend not only on what those who are in front of the group think and do, but also on those who receive the teaching.

Key words: Teacher training, teaching work, good educational practices, quality of education, teacher qualities

\section{Introducción}

Desde que la realidad se constituye como parte de un mundo globalizado, se ha exigido a la ciudadanía ser un ente activo y partícipe en el desarrollo social y económico, a partir de competencias que respondan a las complejas demandas que rodean el mercado laboral internacional, que incluye no sólo el conocimiento científico y desarrollo tecnológico, sino saberes para la sana convivencia y la cultura de la paz.

En la contemporaneidad prevalece la idea de que un mundo progresivo está cimentado en la calidad educativa, por lo que la educación es la base del desarrollo universal (Caballero y Sime, 2006), representa la estructura que viabiliza los altos índices productivos y es pilar de la formación integral de cada sujeto. Esto implica que las escuelas se constituyan como instituciones competentes, que incidan en una interacción sustentada en el mejoramiento colectivo (Molina y Sepúlveda, 2019) para enfrentar las nuevas tendencias, exigencias y paradigmas educativas, a fin de responder a las transformaciones sociales que son cada vez más frecuentes en el siglo XXI, además de que el individuo se integre a esa dinámica mediante la aportación de conocimientos útiles para el avance científico y tecnológico, la solución de problemáticas, el avance de la condición humana y la sostenibilidad.

Ser docente en el contexto de globalización exige una formación integral que permita relacionar la teoría con la práctica en escenarios con particularidades de innovaciones académicas, para una enseñanza que conformen el ente educativo (García, 2020); su rol ha dejado de ser el transmisor de conocimientos, donde reprimía a sus aprendices como medida conductual y cuya opresión del manejo de la clase era inquebrantable, para volverse capaz de contribuir y fomentar actitudes, habilidades y valores que favorezcan al desarrollo escolar. 
No basta con ser eficaz, se requiere ser eficiente y efectivo, pues un profesional apto en la rama de la docencia no es sólo cuestión de conocimientos, sino de saber dominar las cuestiones cognitivas-emocionales, y que, de ellas, se impulsen habilidades personales y profesionales que incluyan vínculos positivos con su labor y con terceras personas que conforman la institución (Herrera et al., 2019). Por lo que cumplir cabalmente con las competencias docentes no garantiza la trascendencia, sino más bien un reconocimiento que puede pasar rápidamente al olvido; para la impronta, se necesita dejar marcas que impulsen la formación continua del alumnado.

Ante ello, ¿en qué consiste la trascendencia en el ámbito educativo?, ¿cómo es posible que el cuerpo docente trascienda entre el estudiantado?, ¿cuáles son las diversas formas en que quien se dedica al magisterio puede trascender? El supuesto del que se parte es que, si el profesorado es activo, sensible a los cambios y está en formación continua, su ejercicio profesional detona su propio desarrollo y deja marcas positivas en la vida y formación de s estudiantes.

El presente artículo se desprende de una investigación cualitativa con enfoque fenomenológico, que tiene el objetivo de analizar la percepción de un grupo de docentes de la Universidad Juárez Autónoma de Tabasco (UJAT) respecto a cómo trascender en y desde su práctica profesional; la exploración del tema surge de la necesidad de conocer si el cuerpo docente está consciente de tales acciones y cómo percibe su desempeño profesional.

\section{Revisión de la literatura}

Competencias para el ejercicio educativo

La Organización de las Naciones Unidas para la Educación, la Ciencia y la Cultura (UNESCO, 2019) identifica al profesorado como: "Una de las fuerzas más sólidas e influyentes con miras a garantizar la equidad, el acceso y la calidad de la educación. Ellos son la clave del desarrollo mundial sostenible”. El hecho de ser docente deja fuertemente una huella en la vida y formación del estudiantado, la cual influirá negativa o positivamente durante su trayectoria académica, puesto que es el principal motor de transformación dentro del ámbito educativo. Como afirma Pogré (2012), la estructura de una sociedad, donde haya una posibilidad de desarrollo equitativo, yace en la transmisión del patrimonio cultural y la enseñanza de los saberes socialmente relevantes, impartida por los adultos dentro de las organizaciones académicas. Ello significa que quien pretende profesionalizarse en esta actividad, deberá desarrollar una serie de competencias que le permita ingresar, permanecer y promoverse en el sistema educativo. 
Considerando el ámbito internacional, la concepción que la Organización para la Cooperación y el Desarrollo Económicos (OCDE, 2019) tiene respecto al trabajo docente es que los profesionales en la materia deben cumplir con las siguientes competencias: 1) Ejecución de las tareas, que incluye la perseverancia, autocontrol, responsabilidad y motivación por lograr resultados; 2) Regulación emocional, que abarca resistencia al estrés, optimismo y control emocional; 3) Colaboración, que engloba la empatía, confianza y cooperación; 4) Mentalidad abierta, que implica curiosidad, tolerancia y creatividad; 5) Relación con los demás, la cual incluye sociabilidad, asertividad y energía; además de la llamada combinación de competencias: pensamiento crítico, metacognición y autoeficacia.

En el contexto nacional, la Nueva Escuela Mexicana (SEP, 2019) establece que quienes imparten clases en educación básica deben cumplir con los siguientes criterios: 1) preparar el trabajo en el aula para facilitar el aprendizaje de los alumnos, el cual dependerá de una planificación con actividades motivadoras, desafiantes y significativas; 2) facilitar el aprendizaje y la participación de todos su alumnado, a través del fomento de la participación, colaboración, inclusión y una enseñanza activa basada en la didáctica planeada; 3) evaluar el aprendizaje de los alumnos con fines de mejora de los logros educativos; 4) atender a los alumnos en un ambiente de equidad, inclusión, interculturalidad, convivencia armónica, seguro y saludable, en un marco de respeto a los derechos humanos; 5) colaborar en la escuela para propiciar el aprendizaje y desarrollo integral del estudiantado, y para participar en acciones orientadas a la mejora de su práctica y a la de su desarrollo profesional.

Respecto al desempeño docente a nivel superior, Torra et al. (2012) consideran que se estructura a través de las competencias interpersonal, metodológica, comunicativa, de planificación y gestión de la docencia, de trabajo en equipo y de innovación. Estas competencias se correlacionan con las mencionadas por Zabalza (2003), las cuales son los saberes para la planificación, didáctica, comunicación, metodología, relaciones interpersonales y tutoría. Visto desde los ejercicios inclusivos, Fernández (2012) declara que las competencias en la educación superior se desarrollan partiendo de las competencias pedagógico-didácticas, de liderazgo, gestión del aprendizaje cooperativo, investigativas, así como las éticas, sociales e interactivas. Por consiguiente, el diseño y aplicación de prácticas inductivas deben beneficiar las exigencias y problemáticas que surgen, y deben ser dirigidas al proceso educativo de cada estudiante. 
En México existen diversas alternativas de educación superior para la formación magisterial (INEE, 2019; SEP, 2017a). Quienes desean integrarse al Servicio Profesional Docente en educación básica, pueden prepararse en las Escuelas Normales Públicas, en la Universidades Pedagógicas Nacionales (UPN), en los Centros de Actualización del Magisterio (CAM), así como en los programas de Ciencias de la Educación, Docencia, Educación y Pedagogía de las universidades públicas, además de las opciones particulares como Escuelas Normales y Centros Universitarios. La educación básica en México consiste en un tipo de educación gratuita y/o privada, estructurada en 3 niveles: preescolar, primaria y secundaria (SEP, 2017b).

Asimismo, hay formación en el campo de la enseñanza para atender a la población estudiantil de los pueblos originarios, quienes además de las competencias docentes que marcan los programas de estudio, deben conocer las lenguas del grupo cultural al que pertenecen. En México existen Universidades Interculturales que contribuyen a la educación en zonas rurales o alejadas de las zonas metropolitanas, atendiendo mayoritariamente a la población indígena. Ofertan programas como Lengua y Cultura, Desarrollo Rural Comunitario, Comunicación Intercultural, que fomentan la incursión de sus profesionales en el ámbito educativo, generalmente en las zonas apartadas (Dietz y Mateos, 2019).

Por su parte, a quienes les interese desarrollarse en el Servicio Profesional Docente de nivel media superior, deben cumplir con perfiles acordes a las asignaturas de los planes de estudio de los subsistemas que operan en México. El nivel educativo de media superior se imparte después del nivel básico; su finalidad es preparar a sus alumnos para que sean capaces de ingresar al nivel superior (SEP, 2017c); la oferta educativa está conformada por 3 modelos: Bachillerato General, Bachillerato Tecnológico y Profesional Técnico (INEE, 2017).

Los subsistemas de educación media superior son proyectos con objetivos y estructuras curriculares que buscan contribuir el desarrollo individual y social de los jóvenes del país (SEMS, 2008). La Subsecretaría de Educación Media Superior, de la Secretaria de Educación Pública, reconoce los siguientes subsistemas: Centro de Educación Tecnológica Agropecuaria (CBTA), Centro de Educación Tecnológica Forestal (CBTF), Centro de Estudios Tecnológicos del Mar (CETMar) y Centro de Estudios de Aguas Continentales (CETAC); Centro de Bachillerato Tecnológico Industrial y de Servicios (CBTIS), Centro de Estudios Tecnológicos Industrial y de Servicio (CETIS) y Centro de Estudios Científicos y Tecnológicos (CECyTEs); Centro de Capacitación para el Trabajo Industrial (CECATI) e Instituto de Capacitación para el Trabajo 
(ICAT); Colegio de Bachilleres estatales (CBT), Preparatorias Federales y Centros de Educación Media Superior a Distancia y preparatorias abiertas; además, se encuentran el Colegio de Bachilleres (COLBACH), el Colegio Nacional de Educación Profesional Técnica (CONALEP) y el Centro de Enseñanza Técnica Industrial (CETI) (SEP, 2018), tanto en modalidad presencial, semipresencial y a distancia.

En el nivel superior, quienes imparten clases son profesionales de diversas áreas del conocimiento que responden a las características de la malla curricular, aunado a niveles de posgrado que deben poseer. Esto significa que ser docente requiere una formación específica, sin embargo, como se aprecia en los niveles media superior y superior, el profesorado no tiene precisamente una formación integral para dedicarse a la enseñanza, sino que ingresan al servicio con solo tener una licenciatura en cualquiera de las áreas del conocimiento, pero deben capacitarse al respecto para adquirir competencias que exige el ejercicio profesional en el ámbito académico.

Las Instituciones de Educación Superior (IES) que forman docentes, actualizan sus planes y programas de estudio a fin de responder a las exigencias contemporáneas educativas, considerando el contexto pluricultural de un país como México; así, el contenido curricular de una Escuela Normal Pública que se ubica en una zona rural difiere de una ubicada en zona urbana, o bien, con una UPN o con un CAM, diferenciándose además por la filosofía organizacional que les dio origen.

Mientras que la UPN forma profesionales que pueden desempeñarse en los niveles de educación básica, media superior y superior, la Escuela Normal Pública focaliza a sus egresados al ejercicio en básica. Las mallas curriculares de ambos organismos son similares puesto que comparten trayectorias formativas, donde se involucra al estudiantado en lo metodológico, humanístico y desarrollo institucional.

Asimismo, quienes estudian Ciencias de la Educación, Pedagogía o Docencia, se involucran en las áreas humanísticas, pedagógicas, gestión educativa, investigación, vinculación social y práctica docente. El contenido curricular de estas licenciaturas varía de acuerdo a la escuela que la oferta, pero comparten las áreas de conocimiento sobre metodología, docencia, pedagogía, administración y psicología.

Distinta es la realidad de quienes estudian una profesión cuya esencia no es la docencia, pero que pueden ejercerla, porque todas las áreas del conocimiento son enseñables, de lo contrario, no se pudieran formar la amplia gama de profesiones que se ofertan en el mundo; para quienes están en esta situación, se entiende que con la capacitación en el área docente obtendrán las herramientas 
para planear, desarrollar y evaluar actividades de enseñanza. Hay quienes se capacitan y hay quienes no lo hacen; así también hay quienes con capacitación docente no ejercen una práctica que trascienda, y hay quienes sin capacitación desarrollan actividades que marcan al estudiantado.

\section{Enseñanzas que dejan marcas}

Cuando se explora un término tan complejo y de carácter absoluto, se torna difícil la tarea de acuñarle una definición. La palabra trascendencia yace en la línea sutil de lo filosófico, teológico y psicológico, caracterizándose con matices vitales concebidos por las conductas susceptibles que el ser humano marca a partir de sus acciones, a lo largo de su vida, colocándole en un sentido o espacio superior fuera de nuestros sentidos y alcances, de manera que su legado rompe las barreras del plano terrenal existencial y abordan más allá de los limites espacio-tiempo.

El significado de la palabra proviene de trascendere: pasar al otro lado, superar, ir más allá de "hacia.” Por consiguiente, trascendencia significa: paso al otro lado, pasar al otro lado y lo trascendente designa aquello hacia donde se produce el paso, aquello que, para ser accesible y comprensible, requiere un paso al otro lado, lo de más allá, lo contrapuesto: por último, lo que trasciende: aquello que realiza el paso más allá. (Heidegger, 2007, p. 188)

El legado de los actos humanos es preservado a través del tiempo, de modo que se toma como un modelo para las generaciones posteriores; así, la personificación de sus acciones define a la persona. Al respecto, Solla y Graterol anotan que: "En este desplazamiento, el comprender, a través de los múltiples individuos, se convierte en el reflejo del ser. Cuando los seres humanos comparten espacio, tiempo y lenguaje entregan su sentir y visión de mundo” (2013, p. 402); visto desde la perspectiva filosófica, la fenomenología de la trascendencia está sustentada bajo un concepto mundano centralmente problematizado en el ser y tiempo (Muñoz Pérez, 2015). De acuerdo con Schmidt, la trascendencia es complicada porque: "se fundamenta desde lo teleológico, lo ontológico y lo axiológico de la naturaleza humana. La búsqueda de la trascendencia, es una necesidad natural del ser humano consciente en dejar un legado o unas huellas a ser seguidas” (2012, p. 54).

El ser humano es capaz de compartir el conocimiento a la sociedad y diversificarlo para un bien común dentro del entorno mismo. Muñoz considera, a partir de lo planteado por Heidegger, que: "el ser humano no sólo produce artefactos o construye puentes, sino que forma con otros seres humanos su historia, su tiempo y su época o, si se prefiere, lleva a cabo sus proyectos” (2015, p. 107). Así, el mundo trascendental es constituido por las cosas materiales, pero, igualmente, se halla 
el hombre visto como un individuo psicofísico, un hecho o ser humano empírico. El esclarecimiento del hombre y su cuerpo forma una trascendencia que debe ser descrita desde sus factores consustanciales en la fenomenología trascendental (Sánchez y Medina, 2018).

Los actos que una persona realiza pueden ser significativos para otros, de tal forma que se graban en la memoria de esos otros, son retenidos como recuerdos y son conectados con la afectividad; cuando sucede eso, tales actos adquieren el significado de trascendencia; el valor afectivo dado a ese legado tiene un impacto personal que será reconocido durante el transcurso de la vida de quien lo ha tomado y podrá ser compartido con la sociedad, lo que a su vez genera conocimiento que forma parte de la experiencia.

\section{Docentes que trascienden}

Intangiblemente existe una fina y delgada línea que puede desequilibrar la ejecución de la práctica docente, a partir del modo que el profesorado decida desempeñarse; por tanto, las instituciones deberían contar con quienes se exigen cumplir debidamente su labor, de manera que sean actores educativos cuya enseñanza sea fructífera para el estudiantado, a través de la generación de ambientes saludables de aprendizaje, de la aplicación de estrategias acordes al contexto del grupo, de la formación continua y del entendimiento de las necesidades del alumnado.

Con respecto a las cualidades, la lista se torna amplia y puede que hasta rigurosa para cumplirlas, sin embargo, se espera que quien se dedique sea capaz de crear un vínculo trascendente con su alumnado. La responsabilidad del profesorado no solo abarca desde una perspectiva educativa, sino también un ámbito cultural, psicológico y sociológico.

Algunas de las características que se consideran para que el sujeto trascienda a partir de su práctica docente son: perfeccionamiento, susceptibilidad al cambio, interactividad con el alumnado, calidad, liderazgo, creatividad, capacidad de planificar, compromiso, vocación, consejería, dinamismo, cuidado de la imagen, facilidad de palabra, inspirador, eficacia, eficiencia y efectividad.

La trascendencia docente es producto de la articulación efectiva de saberes y prácticas; contribuye a la formación del alumnado y deja marcas en el trayecto formativo. Estas huellas deben ser positivas a pesar de que las acciones realizadas para generar el impacto educativo sean consideradas negativas, es decir, el proceso de aprendizaje puede ser percibido por el estudiantado como positivo o negativo, pero el resultado siempre será positivo. Un docente puede que cumpla 
con todas o algunas de las cualidades arriba mencionadas y utilice métodos que respondan a las características de quienes aprenden (positivo), lo que posibilita mayor rendimiento académico (positivo); es decir, si la enseñanza causa un efecto positivo, provocará un resultado positivo; pero existen también docentes que solo cumplen con algunas de esas cualidades (que el alumnado puede considerar como positivo o negativo), sin embargo, sus métodos también posibilitan un éxito académico (positivo); asimismo, hay quienes no reúnen las cualidades deseables y su práctica tiende a ser represiva e impositiva (negativo), pero les funciona para que el alumnado desarrolle conocimientos como anteriormente sucedía en las escuelas públicas (positivo) a pesar del trasfondo por el que fue sometido.

Se deja en claro que no existirá trascendencia cuando quien enseña es deficiente en su ejercicio laboral, ya que se entendería que el producto de esa práctica no planeada ni significativa es escasa o insuficiente, a menos que se tome como ejemplo de lo que no debería hacerse en el ámbito educativo; lo que sí gana quien es docente con poco o nulo rendimiento académico, son calificativos mal intencionados por parte del estudiantado, como burlas, obscenidades y ridiculizaciones.

Por consiguiente, en este proyecto se entiende la trascendencia como el resultado de las acciones de un ser humano, que impacta positivamente en la forma de ser, pensar y actuar de otros; en este caso, el ejercicio que los docentes realizan con el alumnado, quien, a partir de la enseñanza recibida, los definen como mentores modelos a seguir en la práctica académica, que implica una significación afectiva y reconocimiento prolongado.

\section{Metodología}

Para la obtención del dato se hizo uso del enfoque cualitativo de investigación, que consiste en interpretar la realidad a partir de información proporcionada por los sujetos de estudio, donde quien investiga se posiciona dentro del fenómeno que se analiza y no como alguien externo; este acercamiento a la subjetividad de los y las informantes le permite tener mejor percepción y comprensión de lo que investiga.

Como en este trabajo se busca analizar la percepción del cuerpo docente respecto a la trascendencia educativa, se determinó el diseño fenomenológico para el abordaje de la temática. La fenomenología se fundamenta en el estudio de las experiencias de vida, respecto a un suceso, desde la perspectiva del individuo; consiste en comprender las vivencias de los sujetos de 
investigación, de manera que se descifren las percepciones de su alrededor, entendimiento y realidad (Fuster, 2019). Al realizarse un acercamiento inicial a lo que perciben, sienten e interpretan los sujetos de investigación respecto a su práctica educativa en un momento específico, la investigación tuvo un alcance exploratorio.

\section{Unidades de Análisis}

Este proyecto se realizó en la Universidad Juárez Autónoma de Tabasco (UJAT), ubicada en el sureste de México. Es una institución superior que atiende a una población de 61 mil 676 estudiantes, distribuidos en 58 licenciaturas, 17 especialidades, 31 maestrías, 13 doctorados, además de educación a distancia y actividades extracurriculares (cursos, talleres, diplomados), que se imparten en 12 divisiones académicas (UJAT, 2019), y es considerada la máxima casa de estudios del estado de Tabasco, ya que tiene la mayor producción científica y tecnológica en esa entidad. El ejercicio se realizó específicamente en la División Académica de Educación y Artes (DAEA), donde se imparten las Licenciaturas en Gestión y Promoción de la Cultura, Ciencias de la Educación, Idiomas y Comunicación, además de un programa de Técnico Superior Universitario en Música, Maestría en Gestión Educativa y en Intervención e Innovación de la Práctica Educativa, así como un Doctorado en Educación.

Quienes fueron informantes en este proyecto imparten clases en la Licenciatura en Idiomas de la UJAT, donde, de acuerdo con el portal de la DAEA, están adscritos 74 docentes (UJAT, 2020). Para la selección de los sujetos de investigación se tomó en cuenta la subjetividad de la Presidencia de Academia de dicho programa educativo, a quien se le preguntó: ¿A partir de qué cualidades la institución considera que un docente puede trascender?, y ¿qué docentes realizan actividades con las que se pudiera considerar que están trascendiendo? Se contempló, además, la subjetividad del alumnado inscrito en la Licenciatura en Idiomas, con quien se realizó un sondeo a través de llamadas telefónicas, cuentas personales de WhatsApp y un grupo de Facebook llamado Horarios DAEA (Idiomas) (que está avalado por la Sociedad de Alumnos de la división académica), mediante un listado proporcionado por el área de Control Escolar de la DAEA; las preguntas en cuestión fueron: ¿Qué cualidades debe tener el profesorado que trasciende? ¿A quiénes del cuerpo docente de la Licenciatura en Idiomas consideran como mejores calificados y por qué? Lo anterior se efectuó porque interesaba conocer quiénes, a su concepción, son docentes de calidad. 
De acuerdo con Martínez (2012), la muestra cualitativa “depende del propósito del estudio, de lo que resulta útil para lograrlo, de lo que está en juego, de lo que lo hace verosímil, y en última instancia, incluso de lo que es posible” (p.616). Por lo anterior, se invitaron a 15 docentes que tuvieron mayor mención tanto de la Presidencia de Academia como del alumnado. Asimismo, se consideró la saturación del dato como un criterio secundario para la determinación de la muestra, esto es, pudieron haber sido más o menos de 15 sujetos.

\section{Técnica de recolección}

Se utilizó la entrevista para la recolección de datos, ya que permite “raspar” lo complejo del fenómeno que se estudia, a través del diálogo y la percepción del lenguaje no verbal. Varguillas et al. (2007) la describen como una interacción social entre dos personas sobre un tema, descrita como una conversación personal larga y no estructurada, en la que se recopila información sobre conocimientos, creencias, rituales, de una persona o sobre la vida de una sociedad; de manera que quien conteste, manifieste libremente sus opiniones, actitudes o preferencias respecto al tema objeto de estudio, mientras que quien entrevista comprenderá o interpretará el argumento. Las entrevistas se realizaron durante los meses de septiembre y octubre de 2020.

\section{Categorías}

Con el fin de alcanzar el objetivo planteado, se determinaron las siguientes categorías: 1) Cualidades docentes, para identificar los atributos que el profesorado considera tener en su ejercicio laboral, además de conocer a cuál le asigna mayor importancia; 2) Proceso de enseñanza, con el que se buscó conocer cuáles y cómo son las estrategias que aplican para lograr el objetivo académico; 3) Impacto educativo, a fin de conocer el efecto colateral que consideran deja su ejercicio docente entre el alumnado.

\section{Procesamiento de la información}

La construcción del dato se realizó de manera manual por medio de cuadros donde se engloban las respuestas por preguntas, y las preguntas por categorías; cada respuesta se interpretó considerando palabras clave que indicaran similitudes, diferencias, recurrencias y contradicciones, a fin de identificar analizadores y nudos críticos en la experiencia que compartieron respecto a la intervención que realizan como docentes. 


\section{Resultados}

Del cuerpo docente considerado para la entrevista, se tuvo la participación de 10 sujetos debido a que se identificó la saturación del dato, lo que Martínez (2012) afirma que ocurre cuando la información recabada ha topado un límite, es decir, a pesar de que se adjunten nuevas experiencias, ya no se aportan más elementos a la indagación, pues su propia naturaleza habrá sido concluida.

Se aclara que la capacidad operativa de recolección, factor que Hernández et al. (2014) consideran en la determinación de la muestra, estuvo limitada por el contexto de crisis sanitaria provocada por la COVID-19), lo que impulsó a que la obtención del dato fuera en línea y se respetara el tiempo proporcionado por el profesorado, sobre todo de quienes no tienen vinculación con la comunicación interpersonal con medio interpuesto.

El grupo participante tiene en promedio 20 años de servicio en la UJAT, en áreas de Docencia, Idiomas, Traducción e Interpretación, Lingüística e Investigación; con formación profesional en Idiomas y Ciencias de la Educación; la mayoría son docentes de asignatura (no más de 20 horas a la semana), con base laboral y experiencia fuera del entorno académico. Con oportunidad se les entregó el consentimiento informado. Los resultados se presentan considerando las categorías trabajadas.

\section{Cualidades docentes}

El cuerpo docente coincidió en señalar que el humanismo, responsabilidad y compromiso son cualidades que deben desarrollarse en la práctica de la enseñanza, ya que contribuyen íntegramente al ejercicio educativo. Ante ello, considera que ser docente significa guiar a otro ser humano, en el que haya un encuentro de personalidades para compartir la construcción del conocimiento, lo que representa un compromiso para servir en el ámbito académico a los demás; esto quiere decir que quien se dedica a esta actividad sabe que la enseñanza es el medio que permite a los sujetos tener mayores oportunidades para enfrentar su cotidianidad y desenvolverse con efectividad en el ámbito laboral. Se identifican, por un lado, el compromiso, y por otro, la responsabilidad; el compromiso lo decide en su intención de formar profesionalmente a un sujeto, y la responsabilidad, en la respuesta a la demanda del estudiantado.

Ser un docente es un gran compromiso porque de acuerdo a nuestro perfil, nosotros tenemos que compartir conocimiento, instrucciones, experiencia, enseñanza (...) si tengo ese compromiso y esa responsabilidad de utilizar mi conocimiento en cuanto al dominio de la materia, didácticos, pedagógicos, le estoy marcando un camino al estudiante, entonces al 
muchacho se le facilitan las cosas para cuando se enfrente a su realidad, cuando vaya a buscar un empleo. (Sujeto 3, 17 años de servicio)

Significa una gran responsabilidad porque tienes a cargo el deseo de aprender de muchísimas personas que eligen ese camino y que se quieren dedicar a eso. Ser docente requiere de alguien que tenga la capacidad de atender a las necesidades de los estudiantes, y de generar esos aprendizajes para que los estudiantes se sientan motivados a emprender ese camino. (Sujeto 5, 13 años de servicio)

Lo anterior significa tener ecuanimidad, vocación y una formación profesional que le permitan desarrollar competencias en el ámbito educativo. La ecuanimidad hace referencia a la estabilidad emocional del sujeto para estar en condiciones adecuadas de ejercer la docencia. La vocación implica una entrega completa a lo que se realiza, la pasión de hacer la actividad, de tener seguridad de estar en el área adecuada a sus intereses personales, que sea una inspiración diaria para dar lo mejor de sí como docente.

La profesionalización requiere el tránsito del sujeto en instituciones de formación docente, donde a través de la teoría y la práctica adquiera conocimientos y experiencias para actuar con efectividad en el ámbito académico. Se puede tener formación en las aulas, pero si no se tiene experiencia, es probable que haya limitaciones en el ejercicio profesional, por lo que en la docencia resulta importante el desempeño directo en el aula, donde se vivencien las múltiples problemáticas que acechan a la práctica educativa. Asimismo, se adquiere por medio de la capacitación en diversas áreas que impactan en el proceso de enseñanza, tales como pedagogía, didáctica, psicología, investigación y tecnología educativa.

Tiene que recibir instrucción sobre cómo desarrollarte o cómo trabajar en la profesión, en una escuela; también tiene que ver mucho la vocación, los valores, la preparación continua. La preparación continua es vital; en la universidad te dan las herramientas, las estrategias, te enseñan de enfoques, metodologías, pero bueno, ya cuando sales no queda ahí, no te quedas con sólo eso, tienes que seguir preparándote. ¿Cómo se forma? Necesitas universidad y preparación continua. (Sujeto 4, 18 años de servicio)

Se identifica entonces que la disciplina, ética, humildad, empatía, compromiso, responsabilidad, vocación, disciplina, honestidad y consciencia de su quehacer diario, son cualidades que debe tener un docente, lo que significa un perfil completo para desempeñarse en el ámbito laboral. Algunas de estas cualidades son innatas en el sujeto, otras deben cultivarse, porque se debe estar consciente de que no se sabe ni se tiene todo.

Puede que estés bien preparado, pero también debes de contar con una seria disciplina y una conducta ética porque debes formar a otro ser humano. Tienes que saber prepararte 
previo a cada clase, no llegar en ceros, y en eso incide la disciplina. En cuanto a la conducta ética, es tratar y respetar a las personas que tienes enfrente, y ensañarles a aprender a ser respetuosos. (Sujeto 1, 16 años de servicio)

Si hay un atributo que debe tener todo docente es ser empático, uno también tiene que ser muy honesto; tiene que haber una parte humana dentro de esa misma empatía, responsabilidad, disciplina, sencillez, humildad; no somos perfectos. (Sujeto 8, 12 años de servicio)

Esto es, la docencia debe considerar la parte humana de quienes aprenden porque no solo son sujetos que adquieren conocimientos, sino que tienen realidades distintas, las cuales favorecen y/o desfavorecen al proceso de enseñanza; coincidieron en que la empatía es la cualidad más importante, la cual se fomenta con el entendimiento consciente de ser docente, la pasión con la que se realiza la actividad, la adaptación a los diversos contextos educativos y la comprensión a la subjetividad del alumnado.

El ser empático te puede poner en el lugar de la otra persona, tratamos finalmente con una persona que no nada más llega a sentarse a que se llene de conocimientos; es una persona que siente, piensa y reflexiona. Entonces, la empatía y el ser humano te ayudan a tener esa sensibilidad para identificar las necesidades que sí se tienen que cubrir académicamente, pero también las necesidades que la persona quiere cubrir y que son parte de su propósito profesional. (Sujeto 8, 12 años de servicio)

En la misma línea de los atributos docentes, el grupo de participantes considera que a pesar de que cuentan con algunas de las cualidades, hacen el esfuerzo suficiente para establecer un proceso educativo acertado con el que el alumnado se lleve un aprendizaje significativo; afirman ser éticos, pacientes, motivadores, responsables y disciplinados en su ejercicio. Dedicar a desarrollar y/o reforzar cualidades requiere de una actividad introspectiva profunda en la que se reflexione su ejercicio, asimismo ser una persona de amplio criterio, que tome en cuenta las críticas constructivas para identificar aquellos factores que intensifiquen su rendimiento, y posteriormente, los lleven a la práctica.

Estoy casado con la disciplina y la ética, me han sacado adelante, muy a pesar de haber tenido bastantes dificultades al inicio; son dos cosas que siempre me han sacado a flote y que me han permitido estar presente, y en los momentos más difíciles de la profesión, me han permitido sobrevivir. (Sujeto 4, 18 años de servicio)

El ejercicio planeado no siempre ha favorecido el objetivo educativo, ya que afirman que hay factores como la salud física y emocional, situaciones relacionadas a elementos sociales y económicos, adaptación al sistema educativo, manejo del tiempo, estereotipos plasmados en el 
estudiantado y poca consideración del cuerpo docente a la heterogeneidad del grupo, que impactan de forma negativa en el proceso de enseñanza; sin embargo, cuando se busca la excelencia y se poseen las cualidades necesarias, las probabilidades de no lograr el objetivo con sus estudiantes son mínimas.

Los motivos pudieran ser variados, a veces si el docente enferma, difícilmente va a cumplir adecuadamente, o si está pasando por alguna situación emocional. Somos humanos y por muy preparados que estemos y muy racionales que seamos, a veces las cuestiones emocionales nos sobrepasan. (Sujeto 1, 16 años de servicio)

Si un profesor tiene la instrucción, disciplina, ética, dedicación, vocación y los conocimientos, no hay manera de que no pueda salir adelante, de que no logre el objetivo con sus estudiantes; por supuesto, no es infalible, un profesor jamás puede asegurar por completo el objetivo, pero sí tiene mucho porcentaje de que logre los objetivos. (Sujeto 4, 18 años de servicio)

El cuerpo docente puede sufrir de deslices durante su trayectoria, y aunque se reconoce que no es lo idóneo, porque los estudiantes pueden no adquirir los aprendizajes esperados, toman esas experiencias para reflexionar y mejorar su desempeño, de manera que las conviertan en áreas de oportunidad. Coincidieron en afirmar que han fallado en su quehacer profesional, con actos que pudieran considerarse como errores, tales como la empatía limitada, la impuntualidad y el establecimiento de monólogos al ser docentes que acaparan la voz y el tiempo en la sesión de clases.

He fallado en muchos aspectos, uno a veces no tiene el tiempo de dedicárselo a 40 alumnos, porque tengo otros grupos, tengo otras ocupaciones. Ese tiempo que no he logrado es lo que considero una falla, siempre programo cosas para mis clases que en muchas ocasiones no logro completar debido a otros factores: suspensión de clases, modalidades que no esperábamos, en fin... quizás no sean fallas propiamente mías, pero sí las considero así cuando son mis alumnos los que no están logrando el objetivo que me tracé. (Sujeto 9, 22 años de servicio)

Desempeñarse en auténticos contextos áulicos forjarán experiencias en las que se evidencien las realidades de la enseñanza y necesidades del alumnado, por lo que se requiere de atributos para cumplir con una práctica precisa de adaptación constante a los cambios que la educación genere. Ese desempeño es reflejado en el alumnado cuando se le apoya, creando escenarios más beneficiosos para la enseñanza basada en una participación activa. No obstante, es posible que el logro académico pueda decaer por factores personales y/o por las deficiencias de las cualidades que falten por reforzar. 


\section{Procesos de aprendizaje}

Respecto a las estrategias de enseñanza para lograr experiencias exitosas, el cuerpo docente las determina considerando los enfoques de enseñanza, tales como Task Based Learning (TBL), Presentation Practice and Production (PPP), Focus on Form (FonF) y Focus on FormS (FonFS), con el que además aplica la fase práctica en el estudio de los idiomas; asimismo define estrategias que le ha sido útil a partir del número de estudiantes por grupo, así como los intereses para adaptarse al programa y a la naturaleza de la asignatura. En consecuencia, cada uno utiliza sus propios métodos, con los que ha registrado alto aprovechamiento estudiantil, puesto que monitorean el progreso que sus estudiantes tienen durante el trayecto del curso.

Las estrategias van a depender de los grupos, de los niveles; yo no puedo enseñar a los de décimo igual que a los de primero, no puedo enseñar idiomas igual a los de Educación que a los de Comunicación (...) es una constante adecuación, adaptación. (Sujeto 3, 17 años de servicio)

Tengo que saber la naturaleza de la asignatura, cuáles son los contenidos esenciales que los estudiantes deben de asimilar y por qué es necesario que los asimilen, y sobre todo, hacerles ver cuándo les van a ser útiles. (Sujeto 6, 13 años de servicio)

Asimismo, afirman que la confianza es la herramienta más útil a través de los cuales se fomenta la participación activa en el aula mediante preguntas detonadoras o motivándoles a ser capaces de alcanzar los aprendizajes esperados; también hacen uso de técnicas grupales donde se impulsa el trabajo colaborativo, que además funciona para que entre el estudiantado se identifiquen las competencias que cada quien tiene y las aprovechen para la activación del conocimiento y la socialización.

Una de las claves indispensables es fomentar la participación, independientemente de que tienes que hacer la clase lo más atractiva o que el tema se les haga lo más digerible posible para que participen. También la confianza que les des para cometer errores cuando hablan y enfocarte en lo que dicen, determinar estrategias de corrección para no bajar su nivel de confianza. (sujeto 5, 13 años de servicio)

Se identifica además que se aprovechan las deficiencias de aprendizaje que registra el alumnado para convertirlas en oportunidades, donde se obtenga un aprendizaje significativo, ya que se adaptan las planeaciones con nuevas estrategias de enseñanza, considerando lo que necesita quien presenta la deficiencia; así hay quienes promueven la mentoría, que consiste en asesorar a quien en cuestión educativa requiere un reforzamiento; también están los docentes que destinan 
horas adicionales para la asesoría con estudiantes que necesiten el apoyo, donde aplican estrategias personalizadas; y quienes aplican más ejercicios de reforzamiento en las clases subsecuentes.

Te lo voy a poner al revés, no cómo aprovecho deficiencias sino cómo aprovecho los buenos recursos, las buenas competencias que tienen todos los del grupo, porque cuando tengo alumnos que están muy atrasados, los apoyo yo directamente o trato de que sus compañeros quienes están más aventajados me apoyen, porque ellos se sienten más en confianza cuando están entre pares. (Sujeto 2, 20 años de servicio)

Por consiguiente, el profesorado debe desarrollar sus propios recursos metodológicos para lograr clases exitosas, en las que emplee estrategias para facilitar la enseñanza. Sin embargo, deben ajustar las planeaciones si la situación lo amerita, puesto que se tiende a caer en la monotonía, sin prestar atención a los estilos de aprendizaje de cada estudiante. Así, el cuerpo docente debe aprovechar los medios que tiene a su disposición para que eficazmente el aprendizaje sea mutuo. Además, la promoción de un entorno más crítico, práctico y factible, se solventa en la confianza que se deposita en el grupo mediante técnicas interactivas, por tanto, se espera que prospere el rendimiento estudiantil.

\section{Impacto educativo}

El profesorado es evaluado bajo un marco institucional, pero también se evalúa a sí mismo, con el fin de identificar sus fortalezas y áreas de oportunidad para mejorar su práctica. Se entiende que el proceso de evaluación abarca un aspecto objetivo y otro subjetivo, a partir de exámenes de conocimientos de su área formativa, competencias docentes y planeación, así como del análisis de su práctica, respectivamente.

En ese sentido, los sujetos entrevistados consideraron que el cuerpo docente debe ser examinado y/o examinarse sobre los conocimientos del contenido de la asignatura, la capacidad de interacción con el grupo, responsabilidad, tolerancia, asertividad, paciencia, organización, dedicación palpable, habilidad para planear los tópicos, y competencia sobre metodologías diversas, cuyo factor integrador sean la didáctica y la pedagogía.

Yo creo que todo, no nada más la parte del conocimiento, no nada más la parte de tu didáctica, de tu pedagogía, de tu metodología de enseñanza, de tus estrategias de evaluación, sino que además, se debe evaluar mucho esta parte humana, que es la parte empática, la parte social, la parte de la comunicación con tus estudiantes; mucho tiene que ver con que nos evalúen también esa otra parte que no se evalúa, te preguntan nada más por estrategias, si el profesor sabe de estrategia y metodología, pero no te preguntan si el 
profesor busca la manera de acercarse, de ser empático, de compartir contigo el conocimiento. (Sujeto 2, 20 años de servicio)

Respecto a la trascendencia, consideran que en el ámbito educativo se debe ver en dos perspectivas: la práctica docente y la relación con el alumnado. El primero consiste en cumplir con el propósito de la asignatura y de la institución para alcanzar los aprendizajes esperados entre el alumnado, evidenciados con el avance programático que registra el docente; realizar investigación es ineludible para generar y divulgar conocimientos; formar recursos humanos a través de la docencia, asesoría y dirección de proyectos; así como la capacitación continua. El segundo implica en que el alumnado, con las competencias desarrolladas, sea un profesional en el ámbito laboral y sepa resolver las situaciones que se le presenten; que la práctica docente detone la actualización permanente y la cultura del emprendimiento. En términos generales, el profesorado aspira a ser recordado como profesionales que marcaron la vida del estudiantado.

Trascender en el ámbito educativo es que lo que tú hagas y que ello impacte en tu estudiante; si tú logras tener un impacto en él, va a haber una trascendencia en tu trabajo; es dejar una huella prácticamente, dejar buenas experiencias en los alumnos, que ellos sientan que en verdad contigo aprendieron y avanzaron porque en esa medida te van a recordar en sus vidas. (Sujeto 2, 20 años de servicio)

Trascender es ver el avance de nuestros alumnos hasta lograr su meta y que en el proceso nos recuerden como promotores del conocimiento, que fuimos parte de la construcción de su formación educativa. Se ha dicho que un profesor trasciende porque de las aulas saldrán los profesionistas, científicos, humanistas, lingüistas y ciudadanos que serán el futuro de un país o estado. (Sujeto 10, 25 años de servicio)

Por lo anterior se deduce que el cuerpo docente trasciende mediante los productos que genera en el ámbito educativo, tales como artículos científicos y de divulgación, libros, capítulos de libro, antologías, material didáctico; así como con la asesoría de tesis, la integración de redes de investigación, la participación en tutorías y organización de conferencias. Un punto importante es la parte humana, donde es visto como un sujeto que puede apoyar al estudiantado en situaciones adversas fuera del escenario académico; de igual modo, la oratoria es tomada en cuenta, ya que cuando la explicación es clara y elocuente, el alumnado puede percibir la información de manera precisa.

Quienes fueron entrevistados reconocen que hay estrategias que han sido mal aplicadas o que han sido decididas sin considerar el contexto grupal, lo que les ha dado malos resultados; señalan además que hay estilos de enseñanza que no concuerdan con los principios de la educación 
contemporánea; sin embargo, la mayoría afirma que es posible trascender en el ámbito educativo a pesar de que haya errores en la estrategia de enseñanza y/o que se impartan clases que rompen con los estándares normativos. Esto lo toma el alumnado como un referente para decidir qué hacer y qué no cuando se encuentren en el ámbito laboral. En todos los casos posibles, el docente trascendió.

Se trasciende de cualquier maestro porque del maestro malo voy a aprender a cómo a no dar la clase, aprendo de su práctica y es subjetivo. Entonces, de ese docente que yo considero, de acuerdo a mi criterio, no es buen maestro, por consiguiente, de él estoy aprendiendo, y hay una trascendencia porque me está quedando en la psique de cómo no llevar a cabo las cosas. (Sujeto 3, 17 años de servicio)

Puedes trascender siendo un maestro contraejemplo, es decir, es lo que no debe ser un maestro, los estudiantes pueden decir: de mi maestro me gustó tal cosa, eso no me gustó y no lo voy a hacer. En el malo ejercicio de la docencia uno llega a trascender también con los errores que se cometen o con las cosas que uno deja de hacer. (Sujeto 6, 13 años de servicio)

De igual manera, quienes participaron en este ejercicio consideran que su práctica educativa es efectiva porque cumplen con todas o algunas de estas actividades: elaboración puntual de planeaciones de la asignatura, registro del avance de contenidos temáticos conforme está programado, diseño de material didáctico para facilitar el aprendizaje, realización de sesiones adicionales para asesorías y asistencia puntual a las clases, lo que refleja, por un lado, el compromiso que se disponen tener para con el alumnado y la institución educativa, y por otro, la responsabilidad que implica el ser docente. Finalmente, el mejor referente para decidir si son efectivos como profesores es el alumnado.

Yo considero que cumplo con efectividad el rol docente porque el rol del docente es facilitar, orientar, facilitar a los estudiantes el acercamiento al conocimiento, darles las herramientas, propiciar con las estrategias la intervención oportuna para que ellos adquieran nuevos saberes que con lo que ya traen, puedan sumar y puedan sacar un mayor provecho y que en este caso a nivel profesional se vaya conformando como el perfil de Licenciado en Idiomas con el cual deben egresar. (Sujeto 8, 12 años de servicio)

El desempeño efectivo del profesorado impulsa un mejor rendimiento académico cuando el alumnado se percata que su docente tiene compromiso con las sesiones, de tal forma que al medir el progreso estudiantil, ciertas actitudes, que previamente no estaban desarrolladas, son perceptibles, tales como responsabilidad, entusiasmo, participación, comprensión, habilidades 
productivas y receptivas, pero particularmente, los conocimientos del contenido de la asignatura cuando son ejercidos en el ámbito profesional.

La influencia de mi práctica según lo que he visto es que, influye mucho de mis alumnos que son mucho más responsables. Entonces, yo lo que te puedo decir es que la manera en la cual trabajo a ellos les influye positivamente porque yo llego puntual, cumplo con el contenido, me aseguro de que avancen y monitoreo constantemente los avances de investigación. (Sujeto 2, 20 años de servicio)

Asimismo, atribuyen que, desde la percepción del alumnado, los mejores docentes son quienes muestran interés, empatía, confianza, dedicación, vocación, disciplina, amabilidad, respeto y sencillez hacia sus estudiantes; además de que administran adecuadamente el tiempo, aplican dinámicas didácticas y pedagógicas, desarrollan estrategias y enfoques de aprendizaje considerando el contexto grupal, y permiten la interacción alumno-profesor.

Pongo un balance en la parte didáctica y pedagógica... Entonces, me pongo a la altura del estudiante, yo diría: ¿me gustaría enseñarme a mí mismo nada más con el libro? ¿con diapositivas? Entonces mis clases están basadas en eso, en la práctica real, en la que el estudiante se sienta a gusto, conforme, se sienta libre; pongo mucho en balance esa parte pedagógica que tiene que ver con esa comunión emocional que hay entre maestro y estudiante ¿A qué se refiere? A que tú le das una confianza al estudiante y no significa que te va a faltar el respeto. La confianza y el respeto que le das al estudiante hace que se sienta un poco más seguro porque ahí empieza a ver que puede progresar sin que haya ningún trauma. (Sujeto 7, 26 años de servicio)

En términos generales, la trascendencia, de acuerdo con quienes participaron, se constituye a partir de actos en las que el aporte personal influye en aquellos de quienes lo rodean, esto es, puede impactar positivamente en la memoria de los sujetos por haber depositado conocimiento nuevo o porque puede haber cambiado ciertas actitudes que coadyuven a subsistir y a desarrollarse ante las dificultades de la vida diaria. Así, dejan una marca permanente en la memoria del estudiantado a partir del ejercicio educativo que generan en su enseñanza, interacción respetuosa dentro y fuera del aula, vinculación fuera del contexto académico, además de que el alumnado demuestre en su cotidianidad, los conocimientos aprendidos; sin embargo, reconocen que se necesita formación continua para mejorar el quehacer profesional, desarrollar investigación de impacto social, así como tener más tiempo para reforzar conocimientos de los alumnos. Se reconoce que poseer fielmente atributos que mejoren el rol del docente, no garantiza la trascendencia educativa. 


\section{Discusión}

El quehacer docente representa una oportunidad para transformar la cotidianidad del alumnado, labor que implica habilidades innatas o trabajadas en la formación y la práctica profesional, de tal forma que cuando se ejecuta un ejercicio académico, considerando el contexto educativo, se pueden lograr resultados exitosos, los que a su vez propician huellas positivas en quienes participan durante el proceso de aprendizaje.

La percepción del grupo de docentes participante indica que el desenvolvimiento en la enseñanza, con o sin previas nociones, requiere de una ardua profesionalización que posibilite un desempeño adecuado en el contexto áulico -virtual y/o presencial-; tal apreciación coincide con lo obtenido por Barrientos (2016), quien precisa que el magisterio debe desarrollar capacidades creativas, estratégicas, científicas, emocionales y estéticas, aunado a valores como responsabilidad, respeto, gratitud, justicia, honradez, comprensión de la naturaleza de las relaciones humanas, resolución de conflictos y capacidad de diálogo.

En ese tenor de ideas, se rescata lo expresado por la Presidencia de Academia de la Licenciatura en Idiomas, quien afirma que el compromiso del profesorado tanto con la docencia como con la institución, son factores para trascender; ello implica participar en las comisiones educativas porque son parte de la formación profesional, que repercuten en el entorno laboral y, por ende, en el estudiantado; además, en el contexto de crisis sanitaria, el magisterio se ha adaptado a las nuevas modalidades para impartir clases, lo que se puede considerar como novedoso para la práctica docente en el sector público. En cuanto a la percepción del alumnado que respondió al sondeo, cualidades como dinamismo, responsabilidad, motivación y conocimientos de la profesión, son las que consideran esenciales para la trascendencia docente. Estos datos posibilitan la triangulación de datos, que en palabras de Okuda y Gómez (2005), compete la verificación y el contraste de los datos obtenidos en diversas ocasiones y a través de diferentes procedimientos.

Un hallazgo fue que mientras el cuerpo docente participante y la Presidencia de Academia remarcaron el valor del compromiso como elemento indispensable para una buena práctica educativa, el alumnado lo consideró como una cualidad para la trascendencia, pero no entre las más mencionadas.

De igual manera, parece no variar la lista de rasgos que el cuerpo docente debe cumplir si está comprometido en su formación áulica, independientemente de las diversas disciplinas asignadas en los organismos institucionales. En una investigación de Sgreccia et al. (2019) con 
docentes de Matemáticas, se identificó que el buen profesorado atiende las cuatro P: Persistente, Paciente, Presente y (con) Pasión, cualidades que fueron consideradas por los sujetos informantes, que como se indicó, son de Idiomas. Ante ello, surge la interrogante de si tales características tienen la misma relevancia en la impartición de asignaturas distintas y de nivel educativo diferente, y si son validadas por las instituciones y docentes del mundo.

Asimismo, los resultados indican que el grupo de participantes reconoce que la formación profesional docente impulsa conocimientos, habilidades y destrezas para la adquisición de saberes teóricos que faciliten la ejecución del material que el profesorado debe emplear; lo anterior queda acorde con las competencias que marca la Nueva Escuela Mexicana (SEP, 2019) para el ejercicio magisterial, donde se establece que los docentes de educación en todos los niveles: 1) preparen el trabajo en el aula para facilitar el aprendizaje de los alumnos, el cual dependerá de una planificación con actividades motivadoras, desafiantes y significativas; 2) faciliten el aprendizaje y la participación, a través del fomento de la colaboración, inclusión y una enseñanza activa basada en la didáctica planeada; 3) evalúen el aprendizaje con fines de mejora de los logros educativos; 4) atiendan en un ambiente de equidad, inclusión, interculturalidad, convivencia armónica, seguro y saludable, fomentando la sana convivencia, la seguridad y salud en el aula y en la escuela, en un marco de respeto a los derechos humanos; 5) colaboren en la escuela para propiciar el aprendizaje y desarrollo integral de los alumnos, y participen en acciones orientadas a la mejora de su práctica y a su desarrollo profesional.

Se identifica además que la oratoria es un elemento fundamental que, de acuerdo con investigaciones de Abadía et al. (2015), Fernández y Gonzáles (2012), Espinosa (2014), Casero (2016), Chiara (2014), Sgreccia et al. (2019) y Torra et al. (2012), el alumnado considera como el atributo por excelencia del buen profesorado; sin embargo, quienes fueron entrevistados en este ejercicio, lo omitió. Más allá de preparar las clases, desenvolverse en ellas para emplear los temas, utilizar la pizarra y recursos extras como apoyo didáctico, la riqueza de la narrativa oral demanda una interacción que sea inteligible para la comprensión del nuevo conocimiento; del mismo modo, la profundización de temas requiere de una enseñanza prolongada y estratégica que capture puramente el contenido significativo sin que las clases se tornen tediosas ni estresantes.

Si bien el grupo entrevistado concluyó que hacer una introspección sobre su desempeño evita una enseñanza infructuosa y mejora los resultados educativos, cabría preguntarse si en un contexto 
general el profesorado reflexiona sobre su propia ejecución, ya que parece ser que estas acciones pedagógicas representan una relevancia menor:

La competencia reflexiva no está siendo abordada por los propios docentes que permita mejorar y transformar sus prácticas pedagógicas por cuenta propia, no está siendo vista como parte de su rol como educadores, orientadores del proceso de aprendizaje de sus estudiantes. (Agreda y Pérez, 2020, p. 220)

Por otro lado, la elaboración de proyectos de investigación para emprender estudios que promuevan un cambio social deja un aporte pertinente en el campo científico, puesto que forma el rol de docente-investigador y produce conocimientos. Tal como lo afirma Fernández (2012) sobre competencias investigativas, con el que se obtienen conocimientos prácticos y más cercanos al contexto en que se desarrolla el profesorado, quienes fueron entrevistados remarcaron la importancia de involucrarse en la ciencia, no sólo como divulgadores sino como generadores de conocimiento, con el que se percibe mayor compromiso del cuerpo docente hacia el alumnado.

El estudio realizado pudiera extenderse a la comunidad docente de las otras licenciaturas que se imparten en el escenario de investigación, es decir, de la DAEA, porque comparten las mismas características como centro de trabajo y las sociodemográficas del alumnado; sin embargo, es importante considerar las particularidades de los programas de estudio porque aunque estén en el mismo espacio académico, mientras Idiomas se enfoca al aprendizaje de las lenguas, los otros programas direccionan al estudiantado a los procesos de comunicación, la enseñanza y la gestión cultural; por lo tanto, los resultados presentados únicamente se pueden aplicar a la experiencia de quienes imparten clases en Idiomas.

Además, el contexto de pandemia pudiera impactar en la concepción que el profesorado tiene sobre la trascendencia académica porque aunque imparten las clases a distancia, el resultado difiere de lo que se obtiene de forma presencial, y ello provoca que el alumnado pudiera no reconocer el esfuerzo que el docente realiza para manejar tecnología, y ante cualquier falla del uso de los dispositivos, el estudiantado tenga una imagen desfavorecida de quien le imparte la clase; lo mismo ocurre con el cuerpo docente, porque aunque sus estrategias de enseñanza estén bien planeadas y fundamentadas, el que se haga a distancia altera la metodología de aplicación.

Se puede afirmar entonces, que la práctica docente es una actividad compleja que posibilita la transformación de las personas, y a la vez, del contexto social, lo que favorece la construcción de una realidad más justa, democrática y equitativa; en ese quehacer se desarrollan dinámicas que 
generan conocimientos e impulsan competencias para responder a las exigencias de la calidad educativa y del mundo globalizado.

\section{Conclusión}

El profesorado de excelencia debe desarrollar y reforzar cualidades de atención efectiva al estudiantado, a fin de generar un entorno activo de enseñanza-aprendizaje, que considera los estilos particulares de aprehensión del conocimiento; asimismo debe ser susceptible a los cambios constantes para evitar la fosilización de su práctica, que le permitan estar acorde a las exigencias educativas y responder al contexto de globalización de la Nueva Escuela Mexicana. Tal panorama posibilita que quien es docente deje huellas en sus aprendices.

Se recalca que la trascendencia es un estado de subjetividad de quienes se involucran en el proceso de enseñanza-aprendizaje; desde la mirada del docente, el alumnado piensa que el profesorado le ha dejado marcas en su formación académica cuando en el ámbito laboral y en la vida diaria aplican los saberes adquiridos en el aula; es ahí donde están en las altas posibilidades de prevalecer en la memoria escolar.

\section{Referencias}

Abadía Valle, Ana Rosa; Bueno García, Concepción; Ubieto-Artur, María Isabel; Márquez Cebrián, María Dolores; Sabaté Díaz, Sarai; Jorba Noguera, Helga \& Pagès Costa, Teresa. (2015). Competencias del buen docente universitario. Opinión de los estudiantes. REDU. Revista de Docencia Universitaria, 13(2), $363 . \quad$ DOI: https://doi.org/10.4995/redu.2015.5453

Agreda Reyes, Alvaro Alcidez, \& Pérez Azahuanche, Manuel Ángel (2020). Relación entre acompañamiento pedagógico y práctica reflexiva docente. Espacios en Blanco. Revista de Educación, 2(30),219-232.[fecha de Consulta 3 de Febrero de 2021]. ISSN: 1515-9485. Recuperado de: https://www.redalyc.org/articulo.oa?id=3845/384563756002

Aparicio-Molina, Carolina, \& Sepúlveda-López, Felipe. (2019). Trabajo colaborativo docente: nuevas perspectivas para el desarrollo docente. Revista Internacional de Investigación en Ciencias Sociales, 15(1), 109-133. DOI: https://dx.doi.org/10.18004/riics.2019.junio.119133

Barrientos Gutiérrez, Pedro. (2016). La naturaleza de la formación docente. Horizonte de la Ciencia, 6(11),169-177. [fecha de Consulta 27 de Enero de 2021]. ISSN: 2304-4330. Recuperado de: https://www.redalyc.org/articulo.oa?id=5709/570960869015 DOI: https://doi.org/10.26490/uncp.horizonteciencia.2016.11.238 
Caballero-Montañez, R., \& Sime-Poma, L. (2006). "Buen o buena docente” desde la perspectiva de estudiantes que han egresado de educación secundaria. Revista Electrónica Educare, 20(3), 1. DOI: https://doi.org/10.15359/ree.20-3.4

Casero-Martínez, Antonio (2016). Deconstrucción del "buen profesor”. Análisis de las características formales y no formales de la docencia universitaria. RELIEVE, 22(2), art. 4. doi: http://dx.doi.org/10.7203/relieve.22.2.9419

Chiara Conidi, Maria. (2014). La relación maestro-alumno y su influencia en el aprendizaje, la actitud y el crecimiento personal del alumno [Trabajo fin de máster, Universidad $\begin{array}{llll}\text { Internacional de } & \text { La } & \text { Rioja, Barcelona] }\end{array}$ https://reunir.unir.net/bitstream/handle/123456789/2976/MariaChiara_Conidi.pdf?sequen $\mathrm{ce}=1$

Dietz, G., \& Mateos, L. (2019). Las universidades interculturales en México, logros y retos de un nuevo subsistema de educación superior. Estudios Sobre Las Culturas Contemporáneas, XXV(49), 163-190.

Espinosa Martín, M. (2014). Necesidades formativas del docente universitario. REDU. Revista de Docencia Universitaria, 12(4), 161-177. doi:https://doi.org/10.4995/redu.2014.5619

Fernández Batanero, José María. (2012). Capacidades y competencias docentes para la inclusión del alumnado en la educación superior. Revista de la educación superior, 41(162), 9-24. Recuperado en 27 de enero de 2021, de http://www.scielo.org.mx/scielo.php?script=sci_arttext\&pid=S018527602012000200001\&lng=es\&tlng=es

Fernández Borrero, M., \& González Losada, S. (2012). El perfil del buen docente universitario. Una aproximación en función del sexo del alumnado. REDU. Revista de Docencia Universitaria, 10(2), 237-249. doi:https://doi.org/10.4995/redu.2012.6106

Fuster Guillen, Doris Elida. (2019). Investigación cualitativa: Método fenomenológico hermenéutico. Propósitos y Representaciones, 7(1), 201-229. DOI: https://dx.doi.org/10.20511/pyr2019.v7n1.267

García-Conislla, María V. (2020). Relación entre la ejecución curricular y el desempeño docente. Investigación Valdizana, 14(2),103-111.[fecha de Consulta 2 de Febrero de 2021]. ISSN: 1994-1420. Recuperado de: https://www.redalyc.org/articulo.oa?id=5860/586063184005

Heidegger, Martin (2007), Principios metafísicos de la lógica, Madrid, Síntesis.

Hernández Sampieri, Roberto; Fernández Collado, Carlos \& Baptista Lucio, María del Pilar. (2014), Metodología de la investigación, México, McGraw-Hill.

Herrera Torres, Lucía, \& Perandones González, Teresa María, \& Sánchez Sánchez, Laura del Carmen (2019). FORTALEZAS PERSONALES Y EFICACIA DOCENTE. International Journal of Developmental and Educational Psychology, 1(1),317-323.[fecha de Consulta 2 
de Febrero de 2021]. ISSN: 0214-9877. Recuperado de: https://www.redalyc.org/articulo.oa?id=3498/349859739033

Instituto Nacional para la Evaluación de la Educación (INEE) (2019). La formación inicial de los docentes de educación básica en México. Recuperado de: https://www.inee.edu.mx/wpcontent/uploads/2019/08/P3B111.pdf

Instituto Nacional para la Evaluación de la Educación (INEE) (2017). Gaceta de la Política Nacional de Evaluación Educativa en México. Recuperado de: https://historico.mejoredu.gob.mx/voces-y-retos-de-los-subsistemas-de-educacion-mediasuperior/\#: :text=La\%20oferta\%20educativa\%20de\%20la,bachillerato\%20tecnol\%C3\%B 3gico\%20y\%20profesional\%20t\%C3\%A9cnico.\&text=Los\%20principales\%20desaf\%C3 \%ADos\%20que\%20enfrenta,del\%20subsistema\%20estatal\%20de\%20Puebla\%E2\%80\%9 4.

Martínez-Salgado, Carolina. (2012). El muestreo en investigación cualitativa: principios básicos y algunas controversias. Ciência \& Saúde Coletiva, 17(3), 613-619. https://doi.org/10.1590/S1413-81232012000300006

Muñoz Pérez, E. (2015). Trascendencia, mundo y libertad en el entorno de Ser y Tiempo de Martín Heidegger. Veritas, 32(32), 95-110. DOI: https://doi.org/10.4067/s071892732015000100005

Okuda Benavides, Mayumi, \& Gómez-Restrepo, Carlos (2005). Métodos en investigación cualitativa: triangulación. Revista Colombiana de Psiquiatría, XXXIV(1),118-124.[fecha de Consulta 18 de Febrero de 2021]. ISSN: 0034-7450. Disponible en: https://www.redalyc.org/articulo.oa?id=806/80628403009

Organización de las Naciones Unidades para la Educación, Ciencia y Cultura (UNESCO). (2019). Docentes. Recuperado de: https://es.unesco.org/themes/docentes

Organización para la Cooperación y el Desarrollo Económicos (OCDE). (2019). El trabajo de la OCDE sobre Educación y Competencias. Recuperado de: https://www.oecd.org/education/El-trabajo-de-la-ocde-sobre-educacion-ycompetencias.pdf

Pogré, P. (2012). Formar docentes hoy, ¿qué deben comprender los futuros docentes? Perspectiva Educacional, 51(1), 45-56. DOI: https://doi.org/10.4151/07189729-vol.51-iss.1-art.73

Sánchez Muñoz, Rubén \& Medina Delgadillo, Jorge. (2018). El cuerpo vivo y la subjetividad trascendental en la fenomenología de Edmund Husserl. Veritas, 40(40), 9-28. DOI: https://doi.org/10.4067/s0718-92732018000200009

Schmidt Ludwig. (2012). El Hombre Como Ser-Trascendente: Una Perspectiva Judeocristiana. Revista de Bioética Latinoamericana, 10(10), 52-99. Recuperado de: http://www.saber.ula.ve/bitstream/123456789/36074/1/articulo4.pdf.pdf 
Secretaría de Educación Pública (SEP). (2019). Marco para la excelencia en la enseñanza y la gestión escolar dirigidas al aprendizaje y el desarrollo de todos los alumnos. Perfil docente, directivo y de supervisión. Recuperado de: https://evaluacion.septlaxcala.gob.mx/docs/docs_dee/PerfilDocenteDirectivoSupervision MEX_2020-2021.pdf

Secretaría de Educación Pública (SEP). (2018). ¿Qué es la SEMS?. Recuperado de: http://www.sems.gob.mx/es_mx/sems/estructura_sems

Secretaría de Educación Pública (SEP). (2017a). Modelo educativo para la educación obligatoria. Recuperado de: https://www.gob.mx/cms/uploads/attachment/file/207252/Modelo_Educativo_OK.pdf

Secretaría de Educación Pública (SEP). (2017b). Aprendizajes clave para la educación integral. Recuperado de: https://www.planyprogramasdestudio.sep.gob.mx/descargables/APRENDIZAJES_CLAV E_PARA_LA_EDUCACION_INTEGRAL.pdf

Secretaría de Educación Pública (SEP). (2017c). La educación media superior en el sistema educativo nacional. Recuperado de: http://www.sems.gob.mx/es_mx/sems/ems_sistema_educativo_nacional\#: :text=El\%20ti po\%20Medio\%2DSuperior\%20comprende,bachillerato\%20o\%20de\%20sus\%20equivalen tes.

Sgreccia, Natalia, \& Cirelli, Mariela, \& Vital, María-Beatriz (2019). Cualidades de profesores en matemática recordados como buenos por futuros profesores en matemática. Revista Iberoamericana de Educación Superior, 10(27),172-193.[fecha de Consulta 8 de Febrero de 2021]. ISSN: Recuperado

de: https://www.redalyc.org/articulo.oa?id=2991/299159574009

Solla, Rosalía, \& Graterol, Nilsa (2013). La alteridad como puente para la trascendencia ética. Telos, 15(3),400-413.[fecha de Consulta 27 de Enero de 2021]. ISSN: 1317-0570. Recuperado de: https://www.redalyc.org/articulo.oa?id=993/99328424008

Subsecretaría de Educación Media Superior (SEMS). (2008). Reforma integral de la educación media superior en México: la creación de un sistema nacional de bachillerato en un marco de diversidad. Recuperado de: http://cecytemichoacan.edu.mx/wpcontent/uploads/PLANEACION/MARCO\%20JURIDICO/Sistema\%20Nacional\%20de\% 20Bachillerato.pdf

Torra, I., de Corral, I., José Pérez, M., Triadó, X., Pagès, T., Valderrama, E., ... Tena, A. (2012). Identificación de competencias docentes que orienten el desarrollo de planes de formación dirigidos a profesorado universitario. Revista de Docencia Universitaria, 10(2), 21-56.

$\begin{array}{ccccccr}\text { Universidad } & \text { Juárez } & \text { Autónoma de } & \text { Tabasco } & \text { (UJAT). } & \text { (2019). } \\ \text { 4to } & \text { Informe } & \text { de } & \text { Actividades } & 2019 . & \text { Recuperado } & \text { de: }\end{array}$


http://www.archivos.ujat.mx/2020/rectoria/4to-

Informe/CuartoInformedeActividades2019.pdf

Universidad Juárez Autónoma de

Tabasco

(UJAT).

(2020).

Profesores Investigadores de la Licenciatura en Idiomas. Recuperado de: http://www.archivos.ujat.mx/DAEA/actualizacion_2016/05\%20Bot\%C3\%B3n\%20Person al\%20Acad\%C3\%A9mico/Profesores\%20de\%20la\%20Lic.\%20en\%20idiomas.pdf

Varguillas Carmona, Carmen Siavil \& Ribot de Flores, Silvia (2007). Implicaciones conceptuales y metodológicas en la aplicación de la entrevista en profundidad. Laurus, 13(23),249262.[fecha de Consulta 2 de Septiembre de 2020]. ISSN: 1315-883X. Recuperado de: https://www.redalyc.org/pdf/761/76102313.pdf

Zabalza, Miguel (2003), Competencias docentes del profesorado universitario: Calidad y desarrollo profesional. Madrid, Narcea. 